\title{
VII. Oscar Beck \\ 1884-1924. Mit dem Umzug nach München auch \\ juristisch etwas weiter nach oben
}

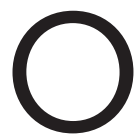

scar Beck war 34 Jahre alt, als Ernst Rohmer ihm 1884, den Verlag übergab. Er hat ihn vierzig lange Jahre bis zu seinem Tod 1924, geleitet, obwohl er als junger Mann viele gesundheitliche Schwierigkeiten hatte. Schon als Schüler auf dem berühmten Wilhelmsgymnasium in München und während der Wehrpflicht in einem Münchener Regiment war er in mehreren Kliniken, danach zu einer längeren Kur in Baden-Baden. Dann hat er 1874, seine Arbeit im Nördlinger Unternehmen begonnen und in den nächsten zehn Jahren alles gelernt, was man als Drucker, Buchhändler und Verleger lernen konnte. 1878 ist er Teilhaber geworden und hat im nächsten Jahr seine Cousine Hedwig Burger geheiratet, Tochter seines Onkels, der Sohn einer Tochter seines Großvaters Carl Heinrich Beck gewesen ist und höherer Beamter der evangelischen Kirche in München.

Der Verleger Oscar Beck war ein Mann, der trotz der zunehmenden Arbeit alles allein machte, auch nachdem er, dem Zug der Zeit im deutschen Buchhandel folgend, 1889 von der Provinz in Nördlingen in die Zentrale nach München zog. Die Druckerei blieb in Nördlingen, bis heute. In München hatte er 1888 vor den Toren der Stadt in der gerade noch selbständigen Gemeinde Schwabing ein Grundstück gekauft und sich ein Verlagsgebäude mit drei Stockwerken bauen lassen, im vornehm bescheide-

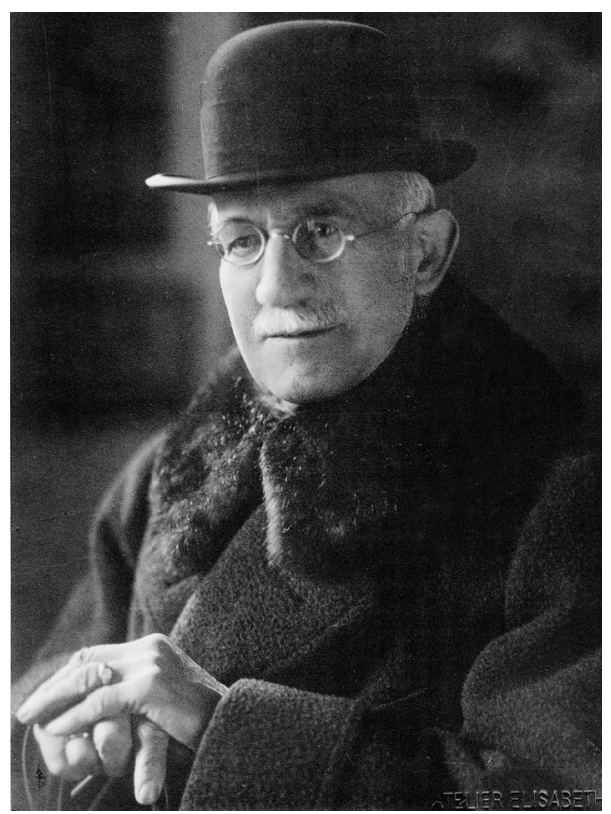

Oscar Beck. Gemäß alter Beschriftung: «Geheimer Kommerzienrat D. Dr. h. C.

Oskar Beck». nen Villenstil, das so genannte rote Palais, mit einem großen Garten. Wie in Nördlingen lebte er hier mit seiner Familie in den beiden oberen Etagen und im Erdgeschoss waren die Räume des Verlags, ein Zimmer für ihn, eins für die technischen Gehilfen, eine Bibliothek und Räume für Papier 

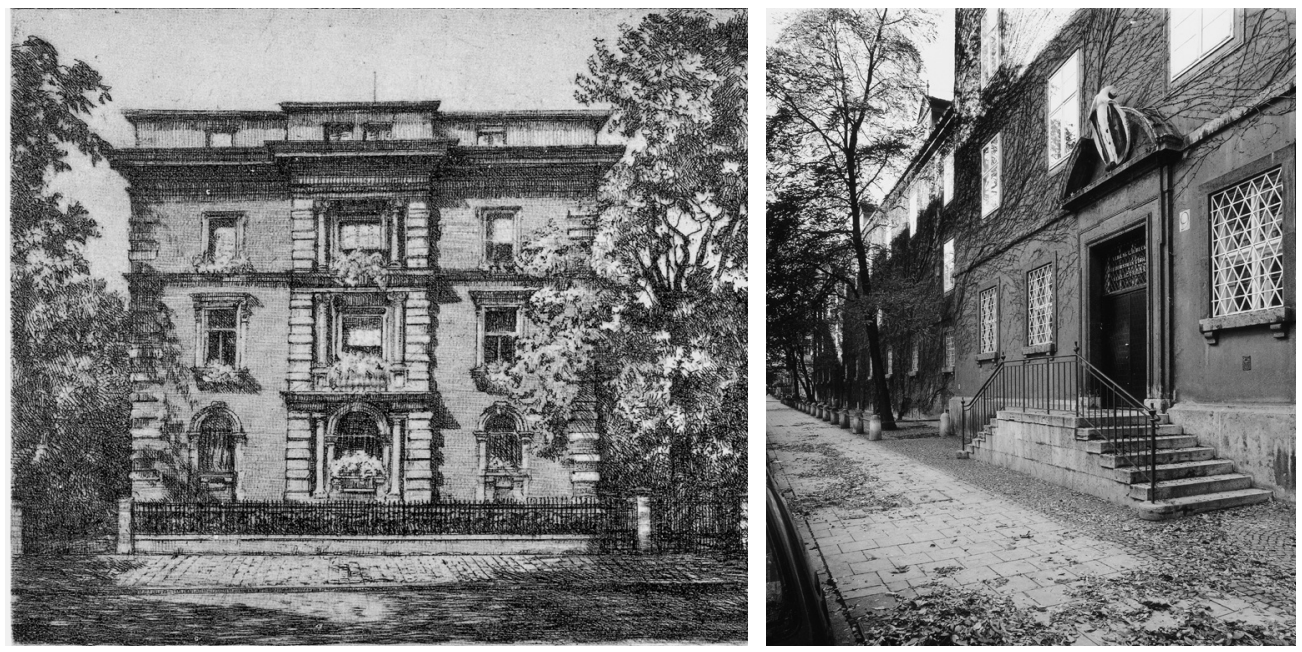

Links das erste Münchener Verlagsgebäude Wilhelmstraße 9, zerstört am 14. Juli 1944. Rechts der neue Bau, im heutigen Sprachgebrauch der «Altbau» mit «Anbau».

und Bücher zur Auslieferung. Trotz der inzwischen größeren Ausweitung des Verlags, führte er die Geschäfte allein, verhandelte selbst mit den Autoren, war sein eigener Lektor, schrieb seine Briefe selbst mit der Hand, wollte weder Sekretärin noch Schreibmaschine, obwohl er ein vermögender Mann war. Das Haus ist 1944 im Zweiten Weltkrieg mit dem Archiv und allem anderen durch Bomben zerstört worden. Sein Sohn Heinrich hat es danach größer wieder aufbauen lassen. Die Adresse aber blieb von 1889 bis heute: Wilhelmstraße 9, Schwabing, inzwischen ein Stadtteil von München.

Den größten literarischen Erfolg hatte Oscar Beck am Ende seiner Arbeit als Verleger. Damals war er schon verzweifelt, weil er wusste, dass der vom deutschen Kaiser und anderen begonnene Weltkrieg das Ende des von ihm geliebten Kaiserreichs sein würde. Aber die Siege am Anfang und die allgemeine Kampfbegeisterung blieben auch auf ihn nicht ohne Wirkung. So hat die Beck'sche Verlagsbuchhandlung in den ersten beiden Kriegsjahren eine größere Zahl von Berichten über die Schlachten der deutschen Truppen veröffentlicht und andere Werke zum Krieg. Als im Osten und Westen der Vormarsch ins Stocken kam, der «Stellungskrieg» begann und die Schrecken des Unternehmens immer deutlicher wurden, endete der Versuch des Verlegers, dem Ganzen doch noch einen Sinn zu geben. Bis auf eine Ausnahme. 
Es war das kleine Buch eines jungen Autors, der den Heldentod für viele deutsche Patrioten ergreifend so gefeiert hat wie Horaz fast zweitausend Jahre vorher in Rom mit dem lateinischen Vers dulce et decorum est pro patria mori, süß und ehrenvoll ist es, für das Vaterland zu sterben. Die Schrift hatte, als Kultbuch in Deutschland noch Wirkung bis zum Ende des «Dritten Reichs» und seinem Zweiten Weltkrieg. Der junge Mann hieß Walter Flex und die knapp einhundert Seiten mit dem Titel «Der Wanderer zwischen beiden Welten» wurde mit später einer Million Exemplaren eines der sechs erfolgsreichsten literarischen Werke zwischen 1915 und 1940 in Deutschland.

Walter Flex, geboren 1887 in Eisenach, Sohn eines Gymnasiallehrers, studierte Germanistik und Geschichte, promovierte 1911 in Erlangen, war von 1910 bis 1913 Hauslehrer der Familie Bismarck, schrieb Novellen und ein Drama über den Reichsgründer, meldete sich 1914, als Kriegsfreiwilliger, wurde 1915 Leutnant der Infanterie und kämpfte in Polen und im Baltikum. Hier entstand die kurze große Freundschaft mit Ernst Wurche, Student der Theologie, ebenfalls Kriegsfreiwilliger und Leutnant im selben Regiment, der im August 1915 bei einem Gefecht mit russischen Truppen getötet wurde. Nach seinem Tod hat Walter Flex ihm ein Denkmal geschrieben auf diesen fast einhundert Seiten mit dem Untertitel «Ein Kriegserlebnis», eine Erzählung, die im Oktober 1916 bei Oscar Beck erschien. Der hatte schon 1915 die ihm von Flex zugeschickte erste kleine Schrift veröffentlicht, «Vom großen Abendmahl. Verse und Gedanken aus dem Felde», knapp

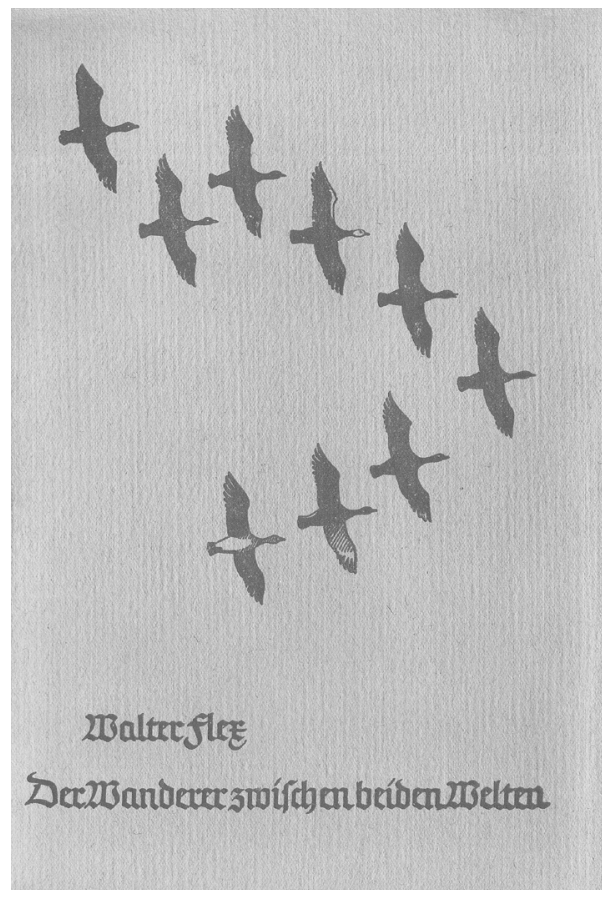

Walter Flex, Der Wanderer zwischen beiden Welten. Wildgänse zieren den Umschlag der Gedächtnisausgabe zum 20. Todestag des am 17. Oktober 1917 gefallenen Autors. 5o Seiten. Wer heute dieses Kriegserlebnis liest, der staunt, wie viel Begeisterung es in den ersten dreißig Jahren bis 1945 gefunden hat mit seinem schwärmerischen Ton und religiösen, vaterländischen und militärischen Übereifer, der den «Sturmangriff» fast in Todessehnsucht verherrlicht als «ewiges Preislied Gottes aus seiner Schöpfung» und die Prosa oft unterbricht mit emotionaler Lyrik, insgesamt drei- 
zehn Gedichte, von denen das erste gleich auf der zweiten Seite gar nicht so schlecht ist, berühmt war und auch mehrfach vertont wurde:

«Wildgänse rauschen durch die Nacht

Mit schrillem Schrei nach Norden -

Unstäte Fahrt! Habt acht, habt acht!

Die Welt ist voller Morden.»

In dieser ersten Strophe schon zwei Ausrufezeichen, in den nächsten drei noch mehr und es endet mit der letzten:

«Wir sind wie ihr ein graues Heer

Und fahr'n in Kaisers Namen,

Und fahr'n wir ohne Wiederkehr,

Rauscht uns im Herbst ein Amen!»

Er wusste nicht, wie Recht er hatte. Es rauschte im Oktober 1917. Da ist er wie sein Freund an jener Ostfront den Heldentod gestorben, war nun auch ein Wanderer zwischen den beiden Welten von Leben und Tod, hatte aber den ersten großen Erfolg seines Buches noch miterlebt. 1917 sind es schon 30000 Exemplare gewesen. Bei Kriegsende 1918 waren es fast 180000. Und so ging es weiter. Als Klassiker im «Dritten Reich» ist es auch für das politische Renommee des Verlags, das durch einen anderen Autor gefährdet war, nicht unwichtig gewesen. Am Ende dieses Reichs und seines Zweiten Weltkriegs waren fast 950 ooo Exemplare verkauft und auch danach ging es noch ein wenig weiter. 1966 war die Million erreicht und 1977 haben die Söhne Heinrich Becks die Rechte an einen kleinen Verlag in der Nähe von Frankfurt am Main verkauft, der damals noch nicht so rechtsextrem gewesen ist wie heute.

Die letzten Jahre wurden schwer für Oscar Beck. Seine Kräfte ließen nach. Dazu kamen neue Krankheiten und die Enttäuschung über die Niederlage im Ersten Weltkrieg. Aber sein Sohn Heinrich hat ihm sofort geholfen. Anfang $1919 \mathrm{kam}$ er als junger Offizier von der «Westfront» nach Hause, mit dem Eisernen Kreuz erster und zweiter Klasse, gab alle Pläne für eine angedachte akademische Karriere auf und übernahm immer mehr die Verantwortung.

Die Lebensleistung seines Vaters Oscar im Vergleich zu der seiner Vorgänger war beachtlich. In den vierzig Jahren als Verleger brachte er es auf etwas mehr als 1 zoo Neuerscheinungen, wenn man die fünf Jahre von 
1919 bis zu seinem Tod Anfang 1924, dazurechnet, in denen die Arbeit im wesentlichen von seinem Sohn Heinrich geleistet worden ist. Das waren im jährlichen Durchschnitt 33, während es bei Carl Beck 25 und Ernst Rohmer 21 gewesen sind.

Die Neuerscheinungen (NE) von Carl Gottlob Beck bis Ernst Rohmer, Zeitschriften nur mit dem ersten Erscheinen.

$\begin{array}{lllllll} & \text { Jahre } & \text { NE } & \text { Jährlicher } & \text { Theolo- } & \text { Schul- } & \text { Juristi- } \\ \text { Verleger } & \text { der } & \text { insge- } & \text { Durch- } & \text { gische NE } & \text { bücher } & \text { sche NE } \\ & \text { Tätig- } & \text { samt } & \text { schnitt } & \text { insgesamt } & \text { insgesamt } & \text { insgesamt } \\ \text { keit } & & \text { der NE } & & & \end{array}$

Carl Gottlob Beck,

Verleger 1763-1802

$29 \quad 132$

4,6

54

8

7

Carl Heinrich Beck,

zunächst als

Unterstützer seiner Mutter als Verlegerin, dann selbst

als Verleger 1802-1834

Catharina Magdalena Beck, Verlegerin als Witwe von Carl Heinrich Beck 1834-1846

Carl Beck, Verleger 1846-1852

Ernst Rohmer, zunächst Unterstützer von Eugenie Beck als Verlegerin, dann als ihr Ehemann Verleger und $32 \quad 11$ 3,5 39 11 6

Statthalter für ihre Söhne mit Carl Beck 1852-1884

Dabei blieb der prozentuale Anteil der juristischen mit einem Drittel der neuen Bücher so groß wie bei Ernst Rohmer. Nur waren es eben sehr viel mehr. Auch Theologie und Schulwesen blieben dahinter weiter Schwerpunkte des Verlags. Das Schulwesen hieß nun Erziehungswissenschaft mit wichtigen Handbüchern. Schulbücher waren nicht mehr dabei. Aber bei Oscar Beck kam noch etwas Neues dazu. Die im 19. Jahrhundert groß 
gewordene deutsche Altertumswissenschaft, klassische Philologie und Alte Geschichte.

Sie erhielten im Verlag Beck eine wichtige Plattform mit dem seit 1886 erscheinenden «Handbuch der klassischen Altertumswissenschaft». Das war zuerst noch in Nördlingen. Herausgeber ist Iwan von Müller gewesen, damals Professor für klassische Philologie (Griechisch und Latein) in Erlangen. Dann begab sich sein Verleger Oscar Beck 1889 nach München und er folgte ihm 1893 mit einer Berufung an die Universität dieser Stadt. Zu Lebzeiten der beiden erschienen ungefähr 40 Bücher, von griechischer Geschichte bis zu lateinischer Grammatik. Das war nicht nur für den Verleger ein wichtiges Unternehmen, das ihm sehr am Herzen lag, sondern ebenfalls für die deutsche Altertumswissenschaft von großer Bedeutung. Ihren hohen internationalen Rang hat sie auch diesem Riesenwerk zu verdanken. Heute sind es im Katalog des Verlags von 1988 sieben eng bedruckte Seiten, darunter für die Rechtsgeschichte die unentbehrlichen Handbücher zum römischen Privat- und Zivilprozessrecht von Max Kaser, inzwischen auch noch zur Geschichte des römischen Rechts von Franz Wieacker und zur Verfassungsgeschichte von Wolfgang Kunkel, den drei großen Männern der Wissenschaft vom römischen Recht in der Bundesrepublik.

In der Reihe der führenden juristischen Verlage hat Oscar Beck noch um 1900 den dritten Platz Ernst Rohmers in Deutschland von 1880 gehalten, obwohl er ein Einzelkämpfer war, anders als Heymanns und Guttentag, und die Gesamtproduktion der rechts- und staatswissenschaftlichen Werke in dieser Zeit fast um mehr als vierzig Prozent gestiegen ist, nämlich von 1829 auf 2598. Hier die Zahlen für 1900:

Führende Verlage nach Titelproduktion 1900

$\begin{array}{llcc}\text { Nr. } & \text { Verlag } & \text { Titel } & \text { Prozent } \\ \text { 1. } & \text { Heymanns, Berlin } & 109 & 4,4 \\ \text { 2. } & \text { Guttentag, Berlin } & 90 & 3,6 \\ \text { 3. } & \text { Manz, Wien } & 63 & 2,5 \\ \text { 4. } & \text { Beck, Nördlingen/München } & 53 & 2,1\end{array}$


Führende Verlage nach Gesamtseitenzahl

Nr. Verlag

1. Heymanns, Berlin

2. Guttentag, Berlin

3. Beck, Nördlingen/München

4. Hof- u. Staatsdruckerei, Wien

5. Manz, Wien

6. G. Fischer, Jena

7. Schweitzer, München

8. Decker, Berlin
Gesamtseitenzahl

33536

28840

16533

15606

13966

12291

11983

11870

Die Zahlen nach Georg Jäger, Juristischer Verlag, in: Jäger u. a. (Hg.), Geschichte des deutschen Buchhandels im 19. und 20. Jahrhundert, Das Kaiserreich 1871-1918, Teil 1, 2001, S. 491.

In diesen Zahlen sind es für Beck in erster Linie die roten Textausgaben oder kurzen Kommentare gewesen. Die erschienen bei den Berlinern wie in Nördlingen, seit 1889 in München, fast immer zeitgleich mit dem Erlass der Reichsgesetze. Die wichtigsten waren in dieser Zeit ${ }_{1883}^{-89}$ die Bismarckschen Sozialgesetze (Kranken-, Unfall- und Rentenversicherung der Arbeiter), das BGB von 1896, das den größten Anstieg brachte, das neue Handelsgesetzbuch 1897, die neue Zivilprozessordnung und das Gesetz über die Freiwillige Gerichtsbarkeit 1898. Nach 1900 kam noch das neue Urheberrechtsgesetz 1907 dazu und in der Weimarer Zeit die Tarifvertragsverordnung 1918, das Betriebsrätegesetz 1920, das Reichsmietengesetz 1922 und 1923 das Mieterschutzgesetz und das Jugendgerichtsgesetz. Aber der Verlag publizierte auch eine umfangreiche Gesetzessammlung, die das bayerische Landes- und das Reichsrecht zusammenfasste: die von Karl Weber seit 1880 herausgegebene «Neue Gesetz- und Verordnungssammlung für das Königreich Bayern mit Einschluß der Reichsgesetzgebung.» Sie umfasste immerhin 42 Bände und erschien bis 1919 .

In den bei Georg Jäger genannten Zahlen für 1910 erscheint Oscar Beck nicht mehr unter den ersten fünf führenden juristischen Verlagen. Tatsächlich hat er in diesem Jahr nur 35 neue juristische Bücher veröffentlicht, weniger als die dort genannten fünf Konkurrenten. Vielleicht lag das daran, dass er schon damals als Einzelkämpfer etwas erschöpft war: 
Führende Verlage nach Titelproduktion im Jahre 1910

$\begin{array}{llcc}\text { Nr. } & \text { Verlag } & \text { Titel } & \text { Prozent } \\ \text { 1. } & \text { Heymanns, Berlin } & 83 & 3,9 \\ \text { 2. } & \text { Guttentag, Berlin } & 70 & 3,3 \\ \text { 3. } & \text { Vahlen, Berlin } & 61 & 2,9 \\ \text { 4. } & \text { G. Fischer, Jena } & 46 & 2,2 \\ \text { 5. } & \text { Duncker \& Humblot, Leipzig } & 44 & 2,1\end{array}$

Zwei große Erfolge und zwei Niederlagen hatte Oscar Beck mit Einzelveröffentlichungen zum Reichsrecht. Die erste Niederlage war das zweibändige Lehrbuch des Zivilprozessrechts von Julius Wilhelm Planck, Professor in München, Vater des Physikers Max Planck und nicht zu verwechseln mit Gottlieb Planck, dem Vorsitzenden der zweiten vom Reichstag eingesetzten Kommission zur Beratung des BGB. Der erste Band erschien 1887, der zweite 1896. Es war ein vorzügliches Buch, erschien sogar 1970 in einem Nachdruck. Aber dann wurde die Zivilprozessordnung von 1877 unter dem Einfluss des BGB 1898 stark verändert, so dass Plancks Lehrbuch schon nach zwei Jahren wieder fast völlig veraltet war. Er ist zu alt gewesen, um eine Neubearbeitung vorzunehmen. 1900 ist er gestorben.

Ebenfalls misslang der Plan eines großen Kommentars zum Bürgerlichen Gesetzbuch, das nach langer Vorarbeit erst 1896 beschlossen wurde, nachdem die Verhandlungen über das Werk schon bald nach $187^{2}$ begonnen hatten, als durch eine Änderung der Reichsverfassung das Parlament auch die Kompetenz erhielt, ein einheitliches Bürgerliches Gesetzbuch für das ganze deutsche Reich zu erlassen. Dann dauerte es aber noch einmal 24. Jahre bis es fertig war und mehrere Autoren, die Oscar Beck zugesagt hatten, am Kommentar mitzuarbeiten, waren entweder gestorben oder zu alt geworden, um ihre Zusagen zu erfüllen. So erschienen nur Bruchstücke, nämlich 1900 der Allgemeine Teil vom Leipziger Professor Eduard Hölder mit 4,8o Seiten, das Allgemeine Schuldrecht von Professor Friedrich Schollmeyer, Universität Würzburg, mit 450 Seiten und 1907/o9 Teile des Familienrechts vom Gießener Professor Artur Schmidt und dem Reichsgerichtsrat August Fuchs mit insgesamt 1300 Seiten. Es blieb ein Torso, der sich trotz großen Lobs für die ersten Teile nicht durchsetzen konnte.

Ein großer Erfolg war dagegen der Kommentar von Otto Fischer und Wilhelm Henle zum BGB, der als einbändige Handausgabe ein Jahr nach dessen Erlass erschienen ist, 1897, lange vor dem Inkrafttreten 1900, eine Seltenheit. Der «Fischer/Henle». Otto Fischer war ein heute fast vergesse- 
ner Jurist von besonderer Art. Zunächst ist er einige Jahre preußischer Amtsrichter gewesen, wurde 1884, Professor in Greifswald und 1890 in Breslau. Ein sehr gescheiter Mann und schon damals eine seltene und heute kaum noch vorhandene Kombination von langer hauptamtlicher Erfahrung in der juristischen Praxis mit danach guter hauptamtlicher wissenschaftlicher Arbeit an der Universität. Wilhelm von Henle war Beamter im bayerischen Innenministerium, um 1900 Regierungsrat, später Ministerialdirektor. Der Erfolg ihres Kommentars war überwältigend. Bis $193^{2}$ hatte er 14, Auflagen mit ungefähr 120000 Exemplaren. Grund dafür war die Auswahl der Bearbeiter. Es sind Praktiker gewesen, die das alte Recht vor dem BGB kannten und nun den Übergang zu den neuen Regelungen deutlich erklären konnten und so dessen «reichsrechtlichen Zuspruch sicherten» (Stefan Saar). Die erste Auflage war ein grauer Leinenband, mit 1150 Seiten schon ziemlich dick und 19 Zentimeter hoch, was man Oktav nennt und zum Beispiel heute die Höhe eines «Schönfelders» ist.

Dieser Fischer/Henle wird ab und zu als

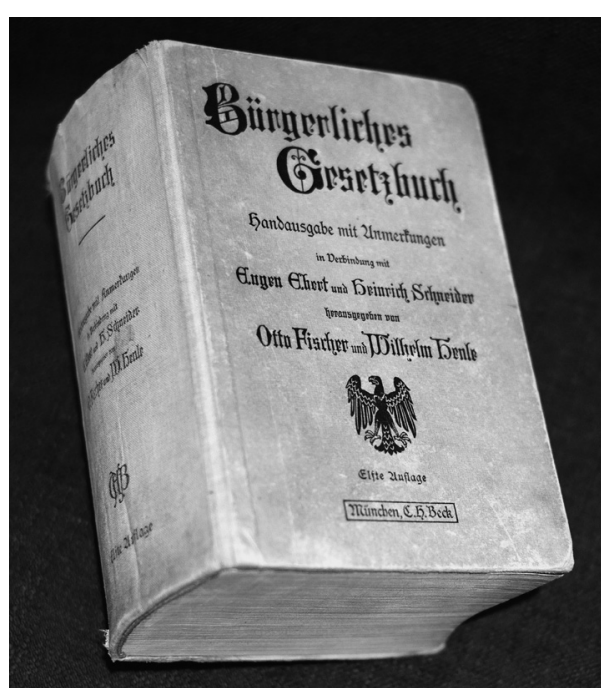

Fischer/Henle, Bürgerliches Gesetzbuch, 11. A. (95. bis 100. Tausend), 1921. Vorläufer des Palandt angesehen. Das ist nicht richtig. Er hatte nämlich eine Schwäche: die so genannte Häkchenmethode. Im Gesetzestext wurden einzelne Worte mit einer hochgestellten Ziffer versehen und dann in dieser Reihenfolge Wort für Wort erklärt. Das mag für Anfänger gar nicht so schlecht sein. Und diejenigen Juristen, die damals als «Anfänger» mit dem BGB arbeiten mussten, haben das offensichtlich auch zu schätzen gewusst. Aber auf die Dauer reichte das nicht. Der Zusammenhang einer Gesetzesvorschrift wird auf diese Weise nicht deutlich genug. Das System, das hinter den einzelnen Worten steht, wird nicht erkannt, und der Sinn dieser Vorschrift erscheint nicht klar genug, durch den der Umgang mit ihr erleichtert wird oder, mit anderen Worten, der eine vollständige und gründliche Interpretation erst möglich macht. Nach dem oft zitierten Satz des römischen Juristen Celsus, der in der Mitte des 2. Jahrhunderts n. Chr. lebte, der «klassischen» Zeit des römischen Rechts, und der bekannt ist für seine markigen Sprüche (1. Buch der Digesten, 3. Titel, 17. Fragment, «Cels.D.1.3.17»): 
«Scire leges non hoc est verba earum tenere, sed vim ac potestatem.» Deutsch: Gesetze zu verstehen heißt nicht, dass man sich an ihre einzelnen Worte klammert, sondern ihren Sinn und Zweck erkennt.

Man nennt das systematische Methode im Gegensatz zu der mit den Häkchen. Das unterscheidet den Palandt vom Fischer/Henle. Auch der Palandt ist ein Kurzkommentar, Handkommentar in einem Band, aber systematisch. Und warum der Fischer/Henle $193^{2}$ mit der 14. Auflage endete und erst 1939 durch den Palandt ersetzt worden ist, wird unten S. 166 ff. beschrieben. Das hatte - leider - auch politische Gründe.

Im Übrigen hat die Ablösung des Fischer/Henle durch den Palandt eine verblüffende Parallele in der mittelalterlichen Geschichte des römischen Rechts. Als die Texte des später so genannten Corpus Iuris Civilis im 11. Jahrhundert gesammelt und erklärt wurden und in Bologna die erste europäische Universität als Rechtsschule entstand mit Irnerius und seinen Nachfolgern, die wir Glossatoren nennen, wurden die Texte dieser damals 5oo Jahre alten Gesetzgebung des oströmischen Kaisers Justinian den Studenten erklärt nach der Methode wie bei Fischer/Henle, nämlich mit der Häkchenmethode, Wort für Wort. Nur mit zwei Unterschieden. Erstens nicht mit Ziffern wie bei Fischer/Henle, sondern mit kleinen Buchstaben. Und zweitens nicht unter dem Text, sondern um ihn herum. Der Text in der Mitte, die Erklärungen am Rand drumherum. Man nennt das Randglossen. Das Wort Glosse ist abgeleitet vom altgriechischen glossa, deutsch Zunge, Sprache, Wort, Erklärung. Im Text hinter den Worten ein hochgestellter kleiner Buchstabe, am Rand mit demselben Buchstaben die Erklärung, Kommentierung.

So blieb es bis zum 14. Jahrhundert. Dann hatte sich diese Methode erschöpft und es kam Bartolus mit seinen Nachfolgern, die wir Postglossatoren oder Kommentatoren nennen. Mit der neuen Methode, wissenschaftlich systematisch wie im Palandt. Nun wurden zum Beispiel in den Digesten, dem wichtigsten Teil der Gesetzgebung Justinians, nicht mehr die einzelnen Fragmente Wort für Wort erklärt, sondern der ganze Abschnitt mit allen Einzelheiten im Zusammenhang, also etwa das Kaufrecht. Bartolus, der berühmteste mit dieser neuen Methode, war noch ein juristischer Doktor in Bologna geworden, danach Professor an den neuen Universitäten in Pisa und Perugia. Weil er der Erste war, galt noch lange der juristische Spruch «nemo jurista nisi Bartolista», deutsch: Niemand ist ein richtiger Jurist, wenn er nicht arbeitet wie Bartolus. 
Der zweite große Erfolg Oscar Becks war der «Sartorius». Im Gegensatz zum Fischer/Henle lebt er bis heute weiter. Carl Sartorius, wurde im Jahre 1865 als Sohn eines Gymnasiallehrers in Bayreuth geboren und war ein Neffe Ernst Rohmers. Er wurde 1901 Professor in Greifswald und 1908 an die berühmte staatswissenschaftliche Fakultät in Tübingen berufen, an der bis zum Ende des Ersten Weltkriegs Gerhard Anschütz, Heinrich Thoma und Rudolf Smend lehrten. Seit 1920 war er Mitglied der liberalen Deutschen Demokratischen Partei, die 1933 wie alle anderen von Hitler aufgelöst wurde, was aber für deren Mitglieder ohne weitere Folgen blieb. Im selben Jahr ist er 68 Jahre alt geworden und wurde emeritiert. Er hat über Verwaltungsrecht, Staatsrecht und Völkerrecht geschrieben und wurde «unsterblich» (Michael Stolleis) durch seine «Sammlung von Reichsgesetzen staats- und verwaltungsrechtlichen Inhalts», die zuerst 1903 bei Beck erschien, als er noch in Greifswald war. Ein kleines rotes Bändchen, Kleinoktav, nur einen halben Zentimeter höher als die normalen roten Textausgaben, mit etwas mehr als 5oo Seiten, zeitlich geordnet, zunächst an der Spitze mit der Bismarckschen Verfassung von 1871, seit 1921 mit der von Weimar 1919, und danach vom Gesetz des Norddeutschen Bundes 1867 über das Passwesen, das Reichs- und Staatsangehörigkeitsgesetz von 1913, den Aufruf des Rats der Volksbeauftragten von 1918 zur Legitimation der neuen Regierung bis zum Gesetz über das Ruhegehalt des Reichspräsidenten von 1922. Seit 1935 ist der «Sartorius» eine Loseblattausgabe, die ständig ergänzt wird, und bis heute neben dem «Schönfelder», der unten S. 93 ff. beschrieben wird, eine der beiden «klassischen» Gesetzessammlungen der Bundesrepublik, inzwischen dick wie ein Ziegelstein, manchmal auch so genannt, und immer noch unentbehrlich für Studium und Praxis.

Nachdem dessen Verkauf so gut lief, dass nach vier Jahren schon eine zweite Auflage gemacht werden musste, hat Oscar Beck 1906 einen entsprechenden Band mit preußischen Gesetzen erscheinen lassen, der einen analogen Titel hatte: «Sammlung preußischer Gesetze staats- und verwaltungsrechtlichen Inhalts», der fast genauso gut verkauft wurde wie der Sartorius. Im Grunde eine erstaunliche Geschichte, dass diese damals wichtigste Sammlung des öffentlichen Rechts in Preußen unter der Herrschaft des bayerischen Prinzregenten Luitpold in München erschien. Herausgeber war Fritz Stier-Somlo, einer der prominenten Professoren des öffentlichen Rechts in der Weimarer Zeit, seit 1904, in Bonn, später in Köln. Das Buch erschien im selben Format wie der Sartorius und in derselben roten Farbe, war nur doppelt so dick, hatte acht Auflagen und erschien 
zum letzten Mal 1934, weil nun die Länder von Adolf Hitler abgeschafft und zu Verwaltungseinheiten des Reichs herabgestuft wurden.

Hatte der Verlag Beck mit der Sammlung von Stier-Somlo auf fremdem Terrain gearbeitet, besaß er im bayerischen Recht schon damals eine dominante Stellung, nicht nur mit Textausgaben, auch bei den Kommentaren. Wie beim Fischer/Henle lag das auch hier an der Auswahl der Autoren, die alle Praktiker gewesen sind. Markus Pollwein, der das bayerische Jagdgesetz kommentierte, war Richter, später am Oberlandesgericht München. Das Buch brachte es von 1885 bis 1928 auf elf Auflagen. Ferdinand von Englert, der den Kommentar zur bayerischen Bauordnung schrieb, war Verwaltungsbeamter, später Präsident der bayerischen Versicherungskammer. Sein Werk brachte es von 1901 bis 1911 auf vier Auflagen und wurde später von anderen weitergeführt. Das dreibändige «Handbuch der inneren Verwaltung» von Wilhelm Krais war mit drei Auflagen im Würzburger Verlag Stuber erschienen und schaffte den Durchbruch 1896/97 mit der vierten, die Oscar Beck übernommen hatte. Wilhelm Krais war damals Vizepräsident des Bayerischen Verwaltungsgerichtshofs in München. Auch die «Blätter für das bayerische Finanzwesen» von 1893 bis 1922 dienten in erster Linie der bayerischen Verwaltungspraxis. Der Umzug nach München hatte den Verlag auch noch mit anderen guten Werken juristisch weitergebracht. 